\title{
Efecto de la Composición Química del Metal de Aporte y del Calor Aportado Sobre la Microestructura y las Propiedades Mecánicas de Juntas Soldadas de Aceros Inoxidables Dúplex
}

\author{
Sebastián Zappa ${ }^{1,2}$, Mónica Zalazar³, Estela Surian ${ }^{4}$ \\ ${ }^{1}$ Universidad Nacional de Lomas de Zamora, Facultad de Ingeniería, Buenos Aires, Argentina. \\ ${ }^{2}$ Consejo Nacional de Investigaciones Científicas y Técnicas - CONICET, Buenos Aires, Argentina. \\ ${ }^{3}$ Universidad Nacional del Comahue, Facultad de Ingeniería, Neuquén, Argentina. \\ ${ }^{4}$ Investigador independiente, Buenos Aires, Argentina.
}

Recibido: 27 Dec., 2016

Aprobado: 05 Jun., 2017

E-mails: zappasebastian@hotmail.com (SZ), monica.zalazar@fain.uncoma.edu. ar (MZ), esurianster@gmail.com (ES)
Resumen: Los aceros inoxidables dúplex poseen una microestructura dual (ferrita y austenita) con contenidos aproximadamente iguales y se caracterizan por tener buena soldabilidad, buenas propiedades mecánicas y una alta resistencia a la corrosión generalizada y localizada. Gracias a estas características, estos aceros son los principales materiales a emplear en cañerías con altas exigencias, ampliamente utilizados en varias industrias, principalmente la petroquímica. Dichas propiedades están controladas por la composición química, el equilibrio microestructural y la ausencia de compuestos intermetálicos. El objetivo de este trabajo fue estudiar la influencia de la composición química del metal de aporte y el calor aportado sobre la microestructura y las propiedades mecánicas en juntas soldadas de cañerías de acero inoxidable dúplex. El metal base utilizado fue un acero inoxidable dúplex UNS S31803 de 8" de diámetro y $8,18 \mathrm{~mm}$ de espesor y los metales de aporte fueron dos alambres tubulares que depositan aceros inoxidables dúplex y lean dúplex (AWS A5.22: E2209T1-1 y E2209T1-G), mediante el proceso de soldadura semi-automático bajo protección gaseosa, soldados con alto y bajo calor aportado. De cada probeta se extrajeron muestras donde se determinó la composición química, se realizó la caracterización microestructural y se determinaron las propiedades mecánicas (dureza, tracción y Charpy-V).

Palabras clave: Acero inoxidable dúplex; Composición química; Soldadura GTAW; Soldadura FCAW; Propiedades mecánicas.

\section{Effect of the Chemical Composition of the Filler Metal and the Heat Input on the Microstructure and the Mechanical Properties of Duplex Stainless Steel Welded Joints}

\begin{abstract}
Duplex stainless steels are characterized by good weldability and mechanical properties; they also present a high resistance to general and localized corrosion. They are the main materials when welding pipes with high requirements in different industries, mainly oil and gas. These steels have a microstructure consisting of two phases: $50 \%$ austenite and $50 \%$ ferrite, approximately, and their properties depend on the maintenance of this ratio and the total chemical composition. The aim of this work was to study the influence of both the chemical composition of the filler metal and the heat input used when welding, on the microstructure and mechanical properties in welded duplex stainless steel pipes. Base metal used was a duplex stainless steel ASTM A790 - UNS S31803 pipe of 8" in diameter and $8.18 \mathrm{~mm}$ in thickness. The filler metals were two tubular wires depositing lean duplex and duplex stainless steels (AWS A5.22: E2209T -1 and E2209T1-G respectively), by semi-automatic welding process under shielding gas, with high and low heat inputs. Samples of each specimen were used to determine chemical composition, to perform the microstructural characterization and to measure mechanical properties (hardness, tensile strength and Charpy-V impact).
\end{abstract}

Key-words: Duplex stainless steel; Lean duplex stainless steel; GTA welding; FCA welding; Mechanical properties.

\section{Introducción}

Los aceros inoxidables dúplex (DSS) son materiales estructurales que presentan una excelente combinación de resistencia mecánica, tenacidad y resistencia a la corrosión. El equilibrio microestructural de ferrita y austenita y el alto contenido de elementos 
Efecto de la Composición Química del Metal de Aporte y del Calor Aportado Sobre la Microestructura y las Propiedades Mecánicas de Juntas Soldadas de Aceros Inoxidables Dúplex

de aleación son los factores más importantes, responsables de la excelente combinación de las propiedades mencionadas [1,2]. Gracias a éstas características son ampliamente utilizados en diferentes industrias, como ser: petroquímicas, nucleares, petróleo y gas, fundamentalmente en plataformas off-shore. Se utilizan con frecuencia en aplicaciones de circulación de soluciones acuosas con altos contenidos de cloruros, reemplazando a los aceros inoxidables austeníticos [3,4].

Durante los últimos años, dentro de la familia de los aceros inoxidables dúplex, se ha desarrollado un nuevo grupo de aceros llamado Lean Duplex Stainless Steels (LDSS), como una alternativa rentable respecto de otros grados de aceros inoxidables. No existe una definición clara de los LDSS pero el término es comúnmente utilizado para designar aceros inoxidables dúplex con bajos contenidos de Ni y Mo. Generalmente, el contenido de Ni se sustituye por una combinación de $\mathrm{Mn}$ y $\mathrm{N}$ y además se agrega Cu para mantener el equilibrio microestructural de la aleación, manteniendo la resistencia mecánica y la resistencia a la corrosión [5,6]. Todos los aceros inoxidables dúplex modernos contienen $\mathrm{N}$ para mejorar las resistencias mecánica y a la corrosión, pero este elemento tiene una implicancia importante respecto de la precipitación de nitruros. La consecuencia de tal precipitación es la pérdida de ductilidad, tenacidad y resistencia a la corrosión. Sin embargo, el $\mathrm{N}$ en solución no sólo aumenta las propiedades mecánicas, sino también mejora la resistencia a la corrosión por picado [2].

Con el fin de mantener una alta resistencia a la corrosión y tenacidad, la zona afectada por el calor (ZAC) de los DSS requiere un contenido de austenita primaria mínimo evitando la formación de $\mathrm{Cr}_{2} \mathrm{~N}$, principalmente en el interior de los granos de ferrita $[7,8]$. Una forma de estudiar dichas transformaciones y precipitaciones es por medio de la medición del tiempo de enfriamiento entre 1200 y $800^{\circ} \mathrm{C}(\Delta \mathrm{T} 12 / 8)$, que es el intervalo de temperatura típico en el que se forma la austenita y se producen precipitaciones en estos aceros $[7,8]$.

Por otro lado, durante la soldadura de los aceros DSS, el recalentamiento impuesto por los cordones sucesivos genera ZAC en donde puede crecer la austenita ya existente o nuclear nueva austenita, llamada austenita secundaria con la precipitación o disolución de nitruros dentro de la ferrita o en las interfaces. Esta nueva austenita secundaria posee un menor contenido de $\mathrm{Cr}$, Mo y N [1]. La austenita secundaria precipita en asociación con la fase sigma por medio de la reacción ferrita $\rightarrow$ austenita secundaria + fase sigma, pudiendo además nuclear en la interfaz $\delta / \delta$ a temperaturas elevadas o en carbonitruros $(\mathrm{Cr} 2 \mathrm{~N})$ dentro de los granos de ferrita. Sin embargo, la austenita secundaria, o sea la austenita formada por medio de un recalentamiento, puede ubicarse en la partícula de austenita ya formada [9]. La precipitación de la austenita secundaria mejora marcadamente la tenacidad de los depósitos [10]. En soldaduras multipasadas, el calor aportado por soldadura y los ciclos térmicos pueden ser controlados para promover la formación de un gran contenido de austenita secundaria. Sin embargo, algunas evidencias indican que la presencia de austenita secundaria puede reducir la resistencia a la corrosión por picado ya que la interface austenita secundaria-ferrita actúa como lugar preferencial de nucleación del pit [10]. Una de las características de esta fase es el bajo contenido de $\mathrm{N}$, lo cual conduce a una menor resistencia a la corrosión $[9,10]$. Este menor contenido de $\mathrm{N}$ en la austenita secundaria podría explicar la menor resistencia a la corrosión por picado en esta fase [10]. Además, los carbonitruros intergranulares favorecen la nucleación de austenita secundaria. Múltiples ciclos de recalentamiento, como los que se encuentran en soldaduras multipasadas, pueden conducir a una muy alta proporción de austenita en el metal de soldadura (MS) [10].

Por otro lado y en términos generales, un enfriamiento demasiado rápido puede generar excesivas cantidades de ferrita y nitruros en todos los aceros inoxidables dúplex a pesar de que la formación de austenita es más rápida en los grados con altos contenidos de nitrógeno. Por otro lado, enfriamiento lento o calentamiento repetido a temperaturas en el rango de aproximadamente $600-1000^{\circ} \mathrm{C}$, pueden generar la formación de fases intermetálicas. La formación de compuestos intermetálicos rara vez es un problema en la soldadura de los DSS o LDSS, mientras que se requiere un control estricto de los procedimientos de soldadura para los grados Superdúplex e Hyperduplex altamente aleados [5].

El objetivo de este trabajo fue estudiar la influencia de la composición química del metal de aporte y del calor aportado sobre la microestructura y las propiedades mecánicas en juntas soldadas de cañerías de acero inoxidable dúplex. 


\section{Procedimiento Experimental}

\subsection{Soldadura}

Se soldaron cuatro juntas de cañerías de aceros inoxidable dúplex, del tipo ASTM A790 - UNS S31803 de 8" de diámetro y 8,18 mm de espesor, mediante los procesos GTAW (soldadura de raíz) y FCAW-G (soldadura de relleno).

Para todas las pasadas de raíz (GTAW) el material de aporte utilizado fue DSS, los gases de protección y de respaldo fueron Ar con un caudal de $15 \mathrm{~L} / \mathrm{min}$ y la posición de las cañerías fija (vertical). Se utilizó un electrodo de tungsteno tipo EWTh-2 de 2,4 mm de diámetro y un stick-up entre 3 y $8 \mathrm{~mm}$. En todos los casos la pasada de raíz fue soldada con idénticos parámetros eléctricos.

Para las pasadas de relleno (FCAW-G), se utilizaron dos metales de aporte DSS [11] y LDSS [12] de 1,2 mm de diámetro. El gas de protección fue $\mathrm{Ar}+20 \% \mathrm{CO} 2$ con un caudal de $18 \mathrm{~L} / \mathrm{min}$, la distancia entre el pico de contacto y la pieza fue de $20 \mathrm{~mm}$ y la posición de soldadura plana bajo mano. Se utilizaron los mismos parámetros eléctricos pero se modificó la velocidad de soldadura para generar dos calores aportados. La Figura 1 muestra el esquema de las juntas realizadas. En la Tabla 1 se muestra la identificación de los cupones y los parámetros de soldadura utilizados. En todos los casos, la limpieza entre cordones fue realizada con cepillos y discos abrasivos para inoxidable.

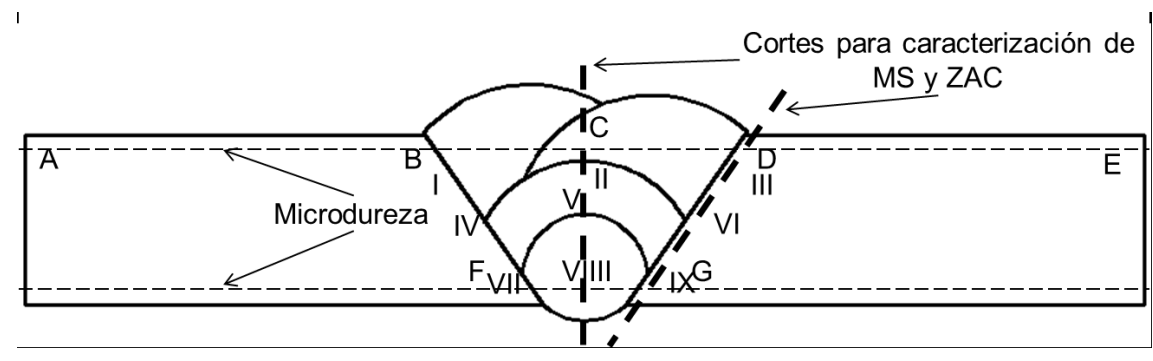

Figura 1. Esquema de diseño de junta y zonas de estudio. Zonas de medición de ferrita mediante ferritómetro: (A y E): metal base; (B, D, F y G): zona afectada por el calor; (C): metal de soldadura. Zonas de medición de ferrita mediante ASTM E562-02: I, III, IV, VI, VII y IX: zona afectada por el calor; II, V y VIII: metal de soldadura.

Tabla 1. Identificación de los cupones y parámetros de soldadura.

\begin{tabular}{|c|c|c|c|c|c|c|c|c|c|}
\hline Cupón & Cordón & Proceso & Aporte & $\begin{array}{c}\text { Gas de } \\
\text { protección }\end{array}$ & $\begin{array}{c}\text { Gas de } \\
\text { Respaldo }\end{array}$ & $\begin{array}{l}\text { Corriente } \\
\text { (A) }\end{array}$ & $\begin{array}{c}\text { Tensión } \\
\text { (V) }\end{array}$ & $\begin{array}{l}\text { Velocidad } \\
\text { (mm/seg) }\end{array}$ & $\begin{array}{c}\text { Calor } \\
\text { aportado } \\
(\mathrm{kJ} / \mathrm{mm})\end{array}$ \\
\hline \multirow{4}{*}{$\mathrm{DH}$} & 1 & GTAW & DSS & $\mathrm{Ar}$ & $\mathrm{Ar}$ & $100-115$ & $8-11$ & 1 & $0,80-1,27$ \\
\hline & 2 & FCAW & DSS & $\mathrm{Ar}+20 \% \mathrm{CO}_{2}$ & N.A. & $135-145$ & $25,0-25,4$ & 3,7 & $0,91-1,00$ \\
\hline & 3 & FCAW & DSS & $\mathrm{Ar}+20 \% \mathrm{CO}_{2}$ & N.A. & $150-170$ & $25,0-25,4$ & 4,1 & $0,91-1,05$ \\
\hline & 4 & FCAW & DSS & $\mathrm{Ar}+20 \% \mathrm{CO}_{2}$ & N.A. & $150-160$ & $25,0-25,2$ & 3,8 & 0,99-1,06 \\
\hline \multirow{4}{*}{$\mathrm{DL}$} & 1 & GTAW & DSS & $\mathrm{Ar}$ & $\mathrm{Ar}$ & $100-115$ & $8-11$ & 1 & $0,80-1,27$ \\
\hline & 2 & FCAW & DSS & $\mathrm{Ar}+20 \% \mathrm{CO}_{2}$ & N.A. & $140-150$ & $25,0-25,1$ & 4,6 & $0,76-0,82$ \\
\hline & 3 & FCAW & DSS & $\mathrm{Ar}+20 \% \mathrm{CO}_{2}$ & N.A. & $140-150$ & $25,1-25,2$ & 4,9 & $0,72-0,77$ \\
\hline & 4 & FCAW & DSS & $\mathrm{Ar}+20 \% \mathrm{CO}_{2}$ & N.A. & $145-155$ & $25,1-25,2$ & 5,1 & $0,71-0,77$ \\
\hline \multirow{4}{*}{ LDH } & 1 & GTAW & DSS & $\mathrm{Ar}$ & $\mathrm{Ar}$ & $100-115$ & $8-11$ & 1 & $0,80-1,27$ \\
\hline & 2 & FCAW & LDSS & $\mathrm{Ar}+20 \% \mathrm{CO}_{2}$ & N.A. & $150-160$ & $24,8-25,5$ & 4,1 & $0,91-1,00$ \\
\hline & 3 & FCAW & LDSS & $\mathrm{Ar}+20 \% \mathrm{CO}_{2}$ & N.A. & $160-170$ & $25,2-25,5$ & 4,5 & $0,90-0,96$ \\
\hline & 4 & FCAW & LDSS & $\mathrm{Ar}+20 \% \mathrm{CO}_{2}$ & N.A. & $170-180$ & $24,9-24,9$ & 3,8 & $1,11-1,18$ \\
\hline \multirow{4}{*}{ LDL } & 1 & GTAW & DSS & $\mathrm{Ar}$ & $\mathrm{Ar}$ & $100-115$ & $8-11$ & 1 & $0,80-1,27$ \\
\hline & 2 & FCAW & LDSS & $\mathrm{Ar}+20 \% \mathrm{CO}_{2}$ & N.A. & $160-170$ & $24,8-25,0$ & 5,4 & $0,73-0,79$ \\
\hline & 3 & FCAW & LDSS & $\mathrm{Ar}+20 \% \mathrm{CO}_{2}$ & N.A. & $150-160$ & $25,0-25,1$ & 5,2 & $0,72-0,77$ \\
\hline & 4 & FCAW & LDSS & $\mathrm{Ar}+20 \% \mathrm{CO}_{2}$ & N.A. & $140-150$ & $25,0-25,1$ & 5,1 & $0,69-0,74$ \\
\hline
\end{tabular}


Efecto de la Composición Química del Metal de Aporte y del Calor Aportado Sobre la Microestructura y las Propiedades Mecánicas de Juntas Soldadas de Aceros Inoxidables Dúplex

\subsection{Inspección visual y radiografía}

Una vez soldadas las juntas se realizaron una inspección visual y radiografía industrial con Iridio 192 y la técnica de doble pared/simple imagen. Los criterios de aceptación de las radiografías fue de acuerdo con el código ASME IX-13 [13].

\subsection{Composición química}

Para determinar la composición química de los materiales de aporte FCAW, se soldaron aportes tipo weldpad con ambos consumibles de acuerdo con SFA 5.22 del código ASME II-13 parte C [14]. Para ello se utilizaron parámetros eléctricos intermedios a los utilizados en las soldaduras de las juntas de cañerías. La medición fue realizada mediante la técnica de espectrometría de emisión óptica en la superficie de dichas probetas.

\subsection{Caracterización macroestructural}

De cada junta soldada se hicieron cortes transversales de $10 \mathrm{~mm}$ de espesor para realizar la caracterización macroestructural a fin de identificar y analizar las diferentes zonas: cañería base (CB), ZAC y MS GTAW y FCAW.

\subsection{Caracterización macro y microestructural}

En diferentes zonas de los cortes transversales se caracterizó la microestructura mediante microscopías óptica (LM) y electrónica de barrido (SEM) y se cuantificaron las diferentes fases mediante ASTM E562-02 [15]. Dicha cuantificación se llevó a cabo en cortes transversales del primer, segundo, tercer y cuarto cuadrante de las cañerías soldadas. Dentro de cada corte transversal, 9 zonas fueron analizadas (zonas I a la IX - Figura 1), con una magnificación de 200x en una grilla de 100 puntos.

\subsection{Medición de ferrita delta}

Se midió el contenido de ferrita en diferentes zonas de las juntas soldadas (zonas A a G - Figura 1) mediante un ferritómetro Fischer Feritscope ${ }^{\circledR}$.

\subsection{Barrido de microdureza Vickers}

Sobre los cortes transversales se realizaron barridos de microdureza Vickers con $1 \mathrm{~kg}$ de carga y $10 \mathrm{~s}$ de aplicación conforme a lo establecido en la norma ASTM E384-11 [16] a 1 mm de la superficies superior e inferior y en el centro de la junta de acuerdo con lo mostrado en la Figura 1. Con estos barridos se midieron las durezas en las zonas de interés: CB, ZAC y MS. Para tener mayor precisión se tomaron mediciones cada 0,5 mm.

\subsection{Ensayos de tracción}

De cada una de las cañerías soldadas se mecanizaron dos probetas de tracción, transversales a la soldadura, extraídas del segundo y cuarto cuadrante, según ASME IX-13 [13].

\subsection{Ensayos de Charpy-V}

Se ensayaron probetas de impacto Charpy-V aplicando la Norma ASTM E-23-2010 [17]. La temperatura de ensayo se fijó en $-53^{\circ} \mathrm{C}$ la cual surge de los requisitos del código ASME VIII Div. 1 (tabla UG 84.2) [18]. Se obtuvieron tres probetas en el MS y tres probetas en la ZAC para cada condición. La Figura 2 muestra la ubicación de la entalla en la ZAC de la soldadura de junta en V. Esto requiere que se realice una macrografía previa a la realización de la entalla. Debido al espesor de la cañería se obtuvieron probetas subsize de $10 \times 5 \times 55 \mathrm{~mm}$.

Los valores de energía absorbida que se consideran aceptables, en la probeta de sección reducida, son de 20J promedio y cualquier valor individual debe ser mayor a 14J. El código ASME VIII UHA - 51 [16], requiere una expansión lateral mayor a 0,38 mm. En los casos donde se obtuvo dispersión en los resultados se analizó la zona de avance de la fractura. 

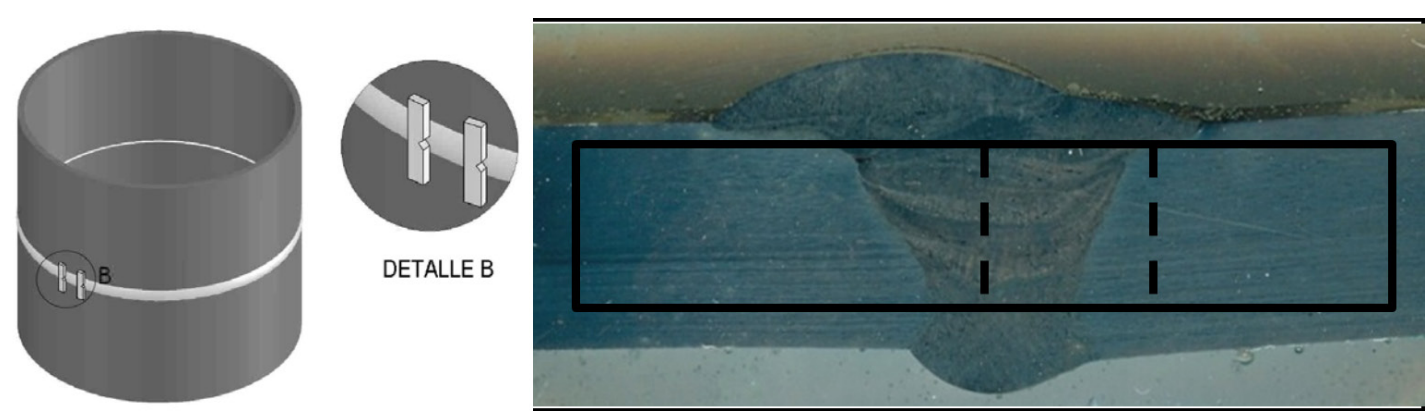

Figura 2. Extracción de las probetas para el ensayo de tenacidad y ubicación de las entallas.

\section{Resultados y Discusión}

\subsection{Inspección visual y radiografía}

Los estudios realizados mediante inspección visual y radiografía mostraron aceptables niveles de indicaciones. Sin embargo, se pudieron observar islas de escoria atrapada en los cupones soldados con consumibles LDSS.

\subsection{Composición química}

La Tabla 2 muestra la composición química obtenida en la cañería y en los metales de aporte puro de ambos consumibles. Además, se presentan los valores suministrados por el fabricante de los consumibles. La técnica de medición utilizada no permitió determinar el porcentaje de N. Se puede observar que tanto las cañerías utilizadas, como los depósitos de ambos consumibles estuvieron dentro de los rangos especificados. En el depósito LDSS se pudo apreciar la disminución del contenido de Mo compensado con el incremento del $\mathrm{Cr}$ (ambos elementos gammágenos).

Tabla 2. Composición química.

\begin{tabular}{|c|c|c|c|c|c|c|c|c|c|}
\hline Muestra & Muestra & $\begin{array}{c}C \\
(\%)\end{array}$ & $\begin{array}{l}M n \\
(\%)\end{array}$ & $\begin{array}{c}\mathbf{S i} \\
(\%)\end{array}$ & $\begin{array}{l}\mathrm{Cr} \\
(\%)\end{array}$ & $\begin{array}{l}\mathrm{Ni} \\
(\%)\end{array}$ & $\begin{array}{l}\text { Mo } \\
\text { (\%) }\end{array}$ & $\begin{array}{c}\mathbf{N} \\
(\%)\end{array}$ & $\mathrm{PRE}_{\mathrm{N}}$ \\
\hline \multirow{2}{*}{ Cañería } & Fabricante & 0,030 & 2,00 & 1,00 & 21,00 & 4,5 & 2,5 & 0,10 & 30,8 \\
\hline & Medido & 0,021 & 1,03 & 0,49 & 21,98 & 5,12 & 3,01 & - & - \\
\hline \multirow{2}{*}{$\begin{array}{c}\text { Consumible } \\
\text { DSS }\end{array}$} & Fabricante & 0,030 & 0,9 & 0,8 & 22,70 & 9,0 & 3,2 & 0,13 & 35,3 \\
\hline & Medido & 0,029 & 1,02 & 0,57 & 22,10 & 8,95 & 2,90 & - & - \\
\hline \multirow{2}{*}{$\begin{array}{l}\text { Consumible } \\
\text { LDSS }\end{array}$} & Fabricante & 0,030 & 0,9 & 0,7 & 24,00 & 9,0 & 0,4 & 0,13 & 27,4 \\
\hline & Medido & 0,030 & 1,07 & 0,56 & 24,67 & 8,76 & 0,15 & - & - \\
\hline
\end{tabular}

PREN (Pitting Resistance Equivalent) $=\mathrm{Cr}+3.3 \times \mathrm{Mo}+16 \times \mathrm{N}[5]$.

La composición química de estos aceros juega un papel muy importante respecto de la resistencia a la corrosión, siendo una de las más afectadas la resistencia al picado. Uno de los métodos más utilizados para conocer la magnitud de la resistencia al picado de los aceros inoxidables es el uso del índice PREN (Pitting Resistance Equivalent Number) $[5,19]$. De acuerdo con la Tabla 2 y con los datos reportados por el fabricante, se puede observar que existen variaciones químicas en los diferentes materiales utilizados que generan distintos comportamientos a la corrosión. Estas diferencias podrían generar diferentes comportamientos frente a la corrosión en diferentes zonas.

\subsection{Caracterización macroestructural}

En la Figura 3 se muestra la macrografía del cupón DH identificando las diferentes zonas: CB, ZAC, MS-GTAW y MS-FCAW y zonas recristalizadas (ZRs). No se observaron defectos macroscópicos, como poros y/o inclusiones. Idénticos resultados se encontraron en los cupones DL, LDH y LDL. En todos los casos, la microestructura estuvo constituida por dos fases, una oscura y una clara, ferrita y austenita respectivamente. Sin embargo, el tamaño, la morfología y el contenido de las fases cambiaron dependiendo de la zona de observación. 
Efecto de la Composición Química del Metal de Aporte y del Calor Aportado Sobre la Microestructura y las Propiedades Mecánicas de Juntas Soldadas de Aceros Inoxidables Dúplex

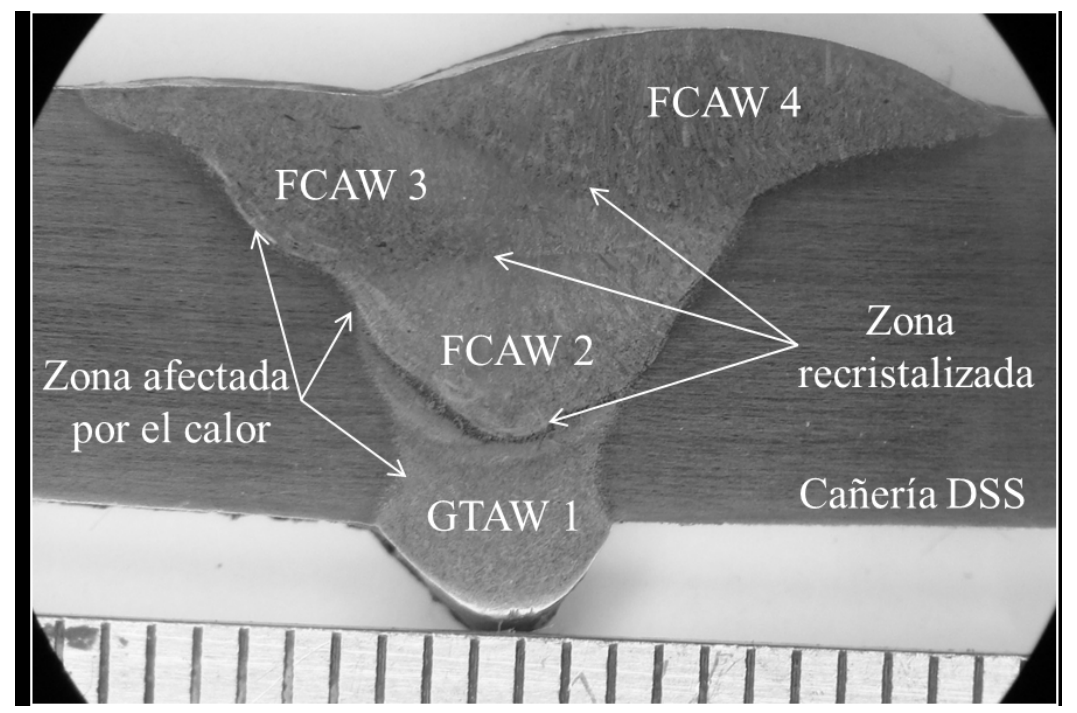

Figura 3. Macrografía del cupón DH.

\subsection{Caracterización microestructural}

La Figura 4 muestra imágenes de LM de las diferentes zonas de la junta DH a diferentes aumentos.

En la Figura 4a se puede observar la microestructura de la CB-DSS con las fases ferrita y austenita alineadas en la dirección de laminación. En la Figura 4b se presenta la ZAC de la CB en la parte inferior de la junta (pasada raíz GTAW). En esta imagen se puede observar la presencia únicamente de ferrita y austenita intergranular y la longitud de la ZAC, que es de tan solo $150 \mu \mathrm{m}$, aproximadamente. La literatura reporta [10] que la ZAC adyacente a la línea de fusión experimenta un recalentamiento que se describe en tres etapas: 1) el material es calentado a muy alta temperatura (sin alcanzar la de fusión). A esta temperatura, la austenita comienza a transformarse en ferrita

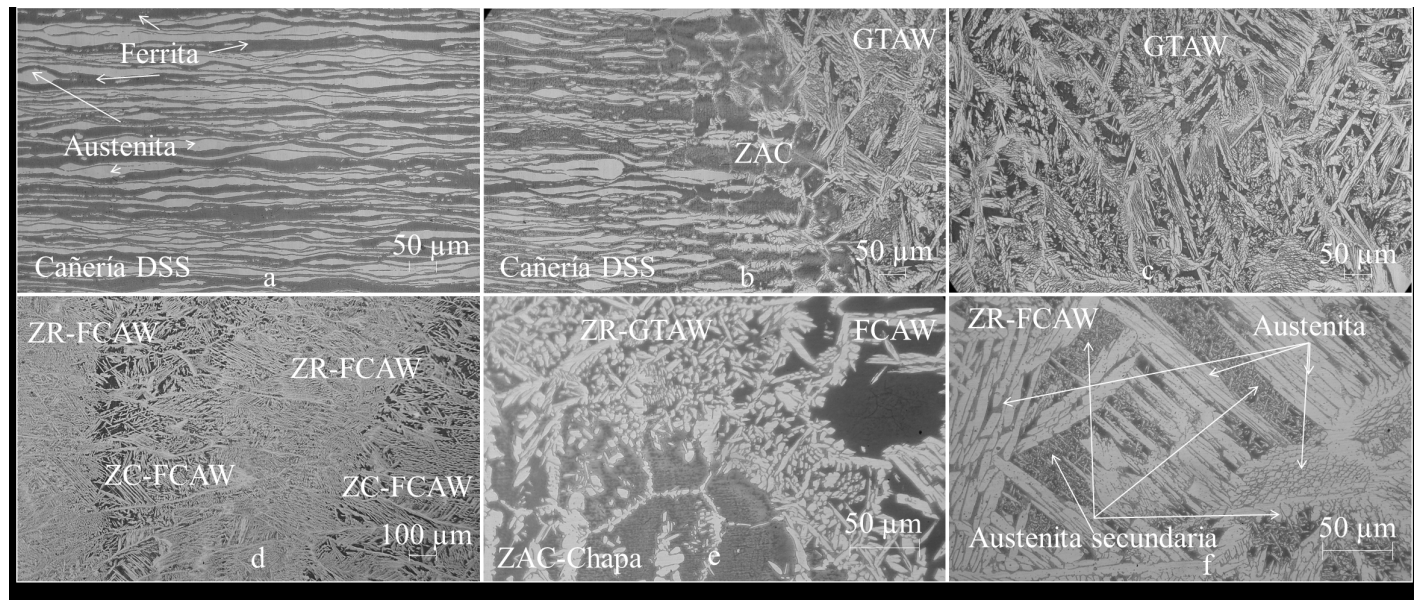

Figura 4. Diferentes zonas de estudio del cupón DH. (a) cañería base; (b) zona afectada por el calor de la cañería base en la parte inferior de la junta; (c) microestructura del cordón GTAW; (d) microestructura de los cordones FCAW; (e) zona donde convergen la zona afectada por el calor, zona recristalizada GTAW y el cordón FCAW; (f) zona recristalizada FCAW.

hasta que, eventualmente, la estructura es completamente ferrítica. En esta instancia, la mayoría de los posibles precipitados (carburos, nitruros, etc.) comienza a disolverse; 2) una vez alcanzada la temperatura pico ocurre el crecimiento de grano de la ferrita, ya que no hay segundas fases o precipitados que lo inhiban; 3 ) durante el enfriamiento, la austenita nuclea y crece, mientras que los precipitados se reforman. La transformación de ferrita a austenita es dependiente de la composición química y de la velocidad de enfriamiento. Con una alta velocidad 
de enfriamiento, no hay tiempo suficiente para la transformación de ferrita en austenita, dando como resultado un alto contenido de ferrita en la ZAC. En estas condiciones de enfriamiento, la precipitación de carburos y nitruros es más pronunciada [10]. Lo discutido anteriormente, justifica el alto contenido de ferrita observado en la ZAC. En la Figura 4c se muestra la microestructura del cordón GTAW, característica del consumible y del proceso. La Figura 4d muestra los cambios generados en la microestructura de los cordones FCAW. En esta imagen se puede observar la zona recristalizada de los cordones de relleno (ZR-FCAW) y la zona columnar (ZC-FCAW). En todos los casos, en las ZRs se encontró precipitación de austenita secundaria, pero la zona inmediatamente por encima de la ZR estuvo caracterizada por un mayor contenido de ferrita, sin observarse precipitación de fases en su interior. El cordón 2 (primer cordón FCAW, ver Figura 1) fue el que mayor contenido de austenita presentó, entre los otros cordones FCAW, ya que este cordón es el que experimenta mayor recalentamiento. La Figura 4e muestra una imagen donde convergen la ZAC, ZR-GTAW y el cordón FCAW. En esta imagen se puede observar la precipitación de austenita secundaria en la ZR-GTAW, asociada al calentamiento sufrido por las pasadas de relleno de la junta. La Figura $4 \mathrm{f}$ muestra ZR-FCAW donde se puede observar austenita Widmanstätten y un gran contenido de austenita secundaria dentro de las placas de ferrita. Finalmente, los cordones superiores FCAW son los que poseen mayores contenidos de ferrita en la estructura. Idénticos resultados se encontraron en las demás juntas soldadas.

La Figura 5 muestra imágenes de SEM del cupón LDL en ZR-FCAW identificándose grandes contenidos de austenita secundaria en el interior de la ferrita.

La literatura [10] reporta que los $\mathrm{Cr}_{2} \mathrm{~N}$ intergranulares son sitios preferenciales para la nucleación de austenita secundaria y puede ocurrir gran cantidad de precipitación durante el recalentamiento en aceros inoxidables dúplex.
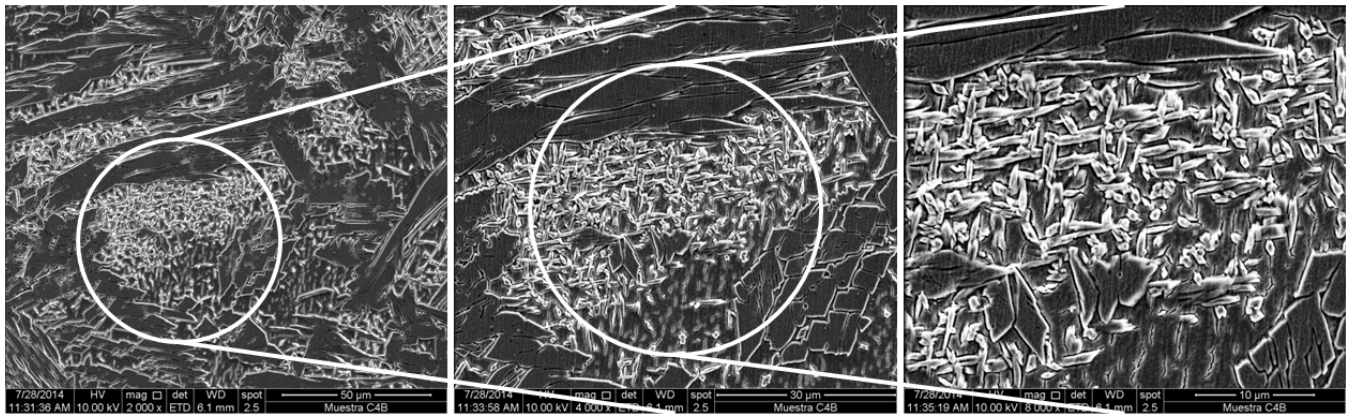

Figura 5. Austenita secundaria en ZR-FCAW del cupón LDL.

Los múltiples recalentamientos, como los experimentados en soldaduras multipasadas, pueden conducir a una gran cantidad de austenita secundaria en el material soldado [10]. Lo discutido anteriormente puede explicar el alto contenido de austenita secundaria mostrado en las Figuras $4 f$ y 5 , indicando la existencia de nitruros de cromo en la microestructura, no identificados con las técnicas utilizadas en este trabajo.

No se observaron variaciones microestructurales mediante LM o SEM en función de la composición química del consumible de relleno ni tampoco en función del calor aportado.

La Tabla 3 muestra los contenidos de ferrita determinados por medio del ferritómetro en diferentes zonas de la junta soldada de acuerdo con la Figura 1. Además se muestran los valores promedio del MS (C) y de la ZAC (B, D, F y G).

Como se observa en la Tabla 3, el contenido de ferrita en todos los casos estuvo comprendido entre un 33 y un $40 \%$.

En la Tabla 4 se presentan los valores promedio de la cuantificación microestructural de ferrita realizada según ASTM E562-02 [15]. Además se muestran los valores promedio del MS (II, V, VIII) y de la ZAC (I, III, IV, VI, VII y IV). De acuerdo con la tabla anterior, se puede observar que en todos los casos los contenidos de ferrita oscilaron de 33 a 56\% en función de la zona analizada. 
Efecto de la Composición Química del Metal de Aporte y del Calor Aportado Sobre la Microestructura y las Propiedades Mecánicas de Juntas Soldadas de Aceros Inoxidables Dúplex

Tabla 3. Contenido de ferrita por ferritómetro.

\begin{tabular}{cccccccccc}
\hline Cupón & $\begin{array}{c}\text { A } \\
(\%)\end{array}$ & $\begin{array}{c}\text { B } \\
(\%)\end{array}$ & $\begin{array}{c}\text { C } \\
(\%)\end{array}$ & $\begin{array}{c}\text { D } \\
(\%)\end{array}$ & $\begin{array}{c}\mathbf{E} \\
(\%)\end{array}$ & $\begin{array}{c}\mathbf{F} \\
(\%)\end{array}$ & $\begin{array}{c}\mathbf{G} \\
(\%)\end{array}$ & $\begin{array}{c}\text { Prom. } \\
\text { MS (\%) }\end{array}$ & $\begin{array}{c}\text { Prom. } \\
\text { ZAC (\%) }\end{array}$ \\
\hline DH & 36 & 33 & 33 & 35 & 34 & 34 & 35 & 33 & 34 \\
DL & 36 & 36 & 36 & 35 & 35 & 37 & 34 & 36 & 36 \\
LDH & 35 & 38 & 35 & 36 & 36 & 37 & 40 & 35 & 38 \\
LDL & 36 & 36 & 37 & 36 & 35 & 39 & 39 & 37 & 38 \\
\hline
\end{tabular}

Si bien las diferencias son pequeñas se observó, de acuerdo con los promedios generales presentados en las Tablas 3 y 4, lo siguiente: 1) las zonas con mayores contenidos de ferrita corresponden a las ZAC, mientras que las zonas con menores contenidos corresponden a los MS. Los mayores contenidos de ferrita en ZAC fueron discutidos anteriormente; 2) los consumibles DSS presentaron menores contenidos de ferrita que los LDSS. Esto podría estar asociado a los mayores contenidos de Cr de estos consumibles respecto de los DSS; 3) mayores contenidos de ferrita en las condiciones soldadas con menor aporte térmico, hecho que podría estar relacionado con las velocidades de enfriamiento, ya que para un menor calor aportado, mayor es la velocidad de enfriamiento y mayor es el contenido de ferrita [5]. Estos resultados cuantifican lo discutido en la caracterización microestructural mediante microscopía óptica. Finalmente, se encontraron diferencias menores de un $10 \%$ entre las técnicas de medición de la ferrita.

Tabla 4. Contenido de ferrita por ASTM E562-02 [15].

\begin{tabular}{cccccccccccc}
\hline Cupón & $\begin{array}{c}\text { I } \\
(\%)\end{array}$ & $\begin{array}{c}\text { II } \\
(\%)\end{array}$ & $\begin{array}{c}\text { III } \\
(\%)\end{array}$ & $\begin{array}{c}\text { IV } \\
(\%)\end{array}$ & $\begin{array}{c}\text { V } \\
(\%)\end{array}$ & $\begin{array}{c}\text { VI } \\
(\%)\end{array}$ & $\begin{array}{c}\text { VII } \\
(\%)\end{array}$ & $\begin{array}{c}\text { VIII } \\
(\%)\end{array}$ & $\begin{array}{c}\text { IX } \\
(\%)\end{array}$ & $\begin{array}{c}\text { Prom. } \\
\text { MS (\%) }\end{array}$ & $\begin{array}{c}\text { Z ZAC (\%) } \\
\text { ZAC }\end{array}$ \\
\hline DH & 50 & 33 & 45 & 45 & 35 & 43 & 49 & 33 & 46 & 34 & 47 \\
DL & 46 & 38 & 53 & 47 & 36 & 44 & 52 & 36 & 46 & 37 & 48 \\
LDH & 50 & 34 & 51 & 50 & 40 & 49 & 46 & 43 & 50 & 39 & 49 \\
LDL & 50 & 36 & 56 & 50 & 34 & 47 & 51 & 46 & 52 & 39 & 51 \\
\hline
\end{tabular}

\subsection{Barridos de microdureza}

La Figura 6 muestra los barridos de microdureza Vickers de cada junta soldada. Dichos valores estuvieron comprendidos entre 220 y $280 \mathrm{HV} 1$. No se encontraron grandes variaciones en las diferentes zonas analizadas (CB, ZAC y MS). Además, se pudo observar en los extremos de los barridos, que la dureza CB fue de 260 HV1, aproximadamente.

Respecto de la dureza en soldaduras DSS, no existe un acuerdo en trabajos anteriores sobre el efecto de las fases. La literatura [20] afirma que la dureza en juntas soldadas está relacionada con los contenidos de ferrita y austenita ya que la ferrita posee una dureza superior y que mayores contenidos de esta fase conllevan a una mayor dureza. Sin embargo otros autores [21,22] aseveran que la dureza de las juntas soldadas no tiene relación con el contenido de fases, al encontrar durezas similares en cordones de soldadura con diferentes contenidos de ferrita. La explicación en este último caso, es que ambas fases poseen composiciones químicas muy cercanas, que generan valores de dureza semejantes. Además otros autores [23] observaron que la dureza de la austenita secundaria es mayor que las de la ferrita y la austenita. Esta observación fue confirmada en el presente trabajo ya que los cordones de pasadas de raíz presentaron mayores contenidos de austenita secundaria y mayor dureza.

De acuerdo con la Figura 6, y observando los barridos de dureza superiores y medios (que tuvieron un comportamiento similar) se observa que la dureza de la cañería base fue mayor que la de los cordones soldados. Además, se notó un muy leve incremento de dureza en la ZAC de la cañería base. Esto podría estar asociado a los mayores contenidos de ferrita en esta zona [20]. Sin embargo, se observó que la dureza de la pasada de raíz 

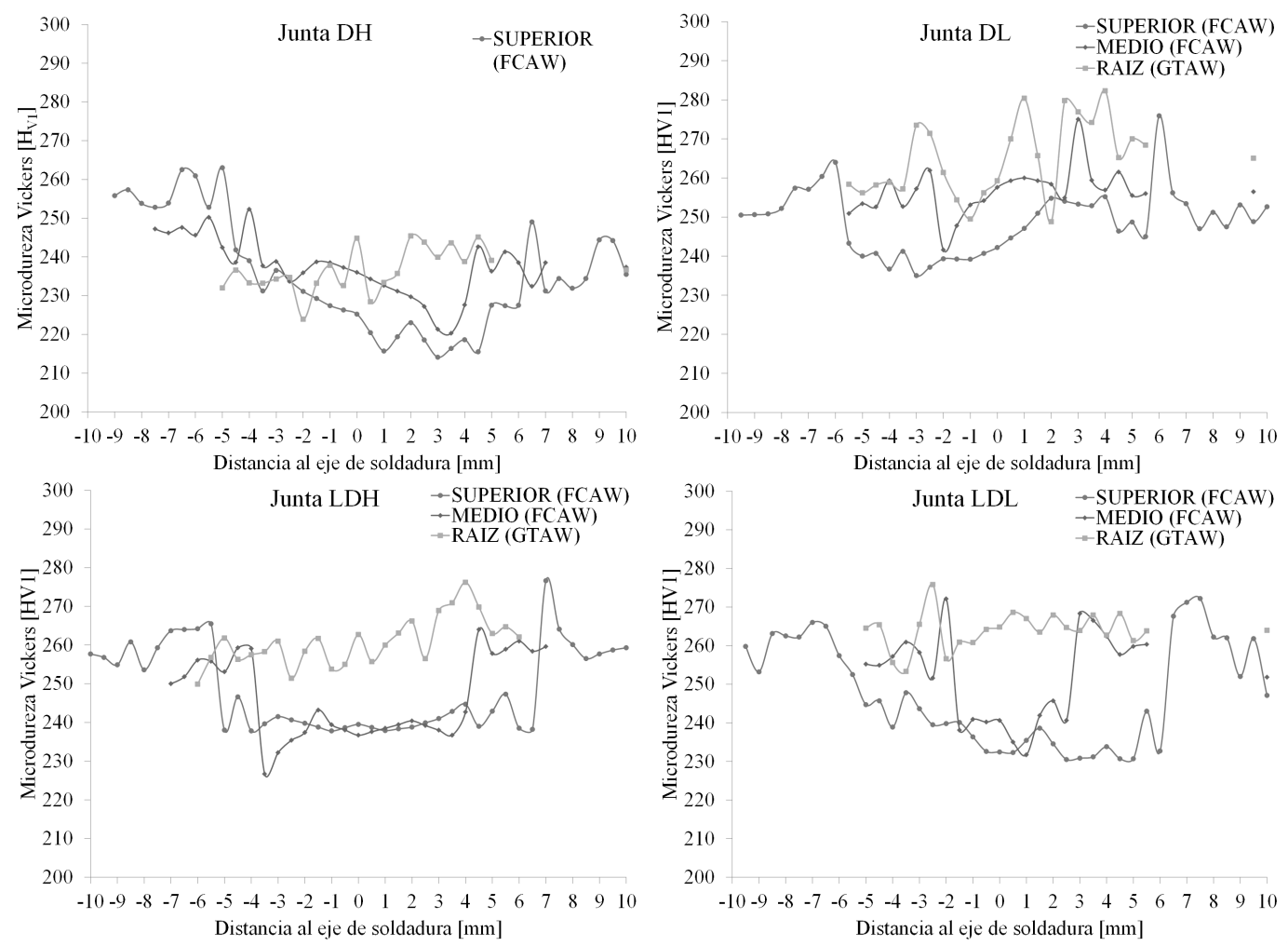

Figura 6. Barridos de microdureza Vickers.

(GTAW) tuvo una leve mayor dureza respecto de los cordones de relleno (FCAW). Esto podría estar asociado al mayor contenido de austenita secundaria encontrado en esta zona [23]. Mayores estudios son necesarios para clarificar el efecto que tienen las diferentes fases respecto de la dureza.

La Tabla 5 muestra los promedios de los barridos de dureza, tanto de la ZAC como del MS y los resultados obtenidos de los ensayos de tracción de todas las condiciones.

De la Tabla 5 se puede concluir que no se encontraron grandes variaciones en los promedios generales de dureza de las distintas zonas. Sin embargo, en términos generales se observó lo siguiente: 1) la dureza de la ZAC fue mayor que la del MS; 2) los MS DSS tuvieron menor dureza; 3) las juntas soldadas con menor calor aportado presentaron mayor dureza. Las justificaciones de los puntos anteriores estarían relacionadas con los contenidos de ferrita discutidos anteriormente.

Tabla 5. Valores promedio de dureza y propiedades en tracción.

\begin{tabular}{|c|c|c|c|c|c|c|}
\hline Cupón & $\begin{array}{l}\text { Dureza MS } \\
\text { (HV1) }\end{array}$ & $\begin{array}{c}\text { Dureza ZAC } \\
\text { (HV1) }\end{array}$ & $\begin{array}{c}\text { RT } \\
\text { (MPa) }\end{array}$ & $\begin{array}{c}\mathbf{R}_{0,2} \\
(\mathrm{MPa})\end{array}$ & $\begin{array}{c}E \\
(\%)\end{array}$ & $\begin{array}{l}\text { Zona de } \\
\text { fractura }\end{array}$ \\
\hline $\mathrm{DH}$ & 232 & 245 & $\begin{array}{l}834 \\
822\end{array}$ & $\begin{array}{l}665 \\
652\end{array}$ & $\begin{array}{l}28 \\
27\end{array}$ & $\begin{array}{l}C B \\
C B\end{array}$ \\
\hline $\mathrm{DL}$ & 246 & 272 & $\begin{array}{l}840 \\
844\end{array}$ & $\begin{array}{l}716 \\
663\end{array}$ & $\begin{array}{l}21 \\
23\end{array}$ & $\begin{array}{l}C B \\
C B\end{array}$ \\
\hline LDH & 246 & 267 & $\begin{array}{l}774 \\
774\end{array}$ & $\begin{array}{l}667 \\
657\end{array}$ & $\begin{array}{l}7 \\
8\end{array}$ & $\begin{array}{l}\text { MS } \\
\text { MS }\end{array}$ \\
\hline LDL & 247 & 264 & $\begin{array}{l}861 \\
831\end{array}$ & $\begin{array}{l}668 \\
620\end{array}$ & $\begin{array}{l}26 \\
22\end{array}$ & $\begin{array}{l}C B \\
C B\end{array}$ \\
\hline
\end{tabular}

Siendo MS: metal de soldadura; ZAC: zona afectada por el calor; RT: Resistencia a la tracción; $R_{0,2}$ : Resistencia a fluencia; E: elongación; CB: cañería base. 
Efecto de la Composición Química del Metal de Aporte y del Calor Aportado Sobre la Microestructura y las Propiedades Mecánicas de Juntas Soldadas de Aceros Inoxidables Dúplex

\subsection{Ensayos de tracción}

Respecto de los resultados obtenidos de los ensayos mecánicos presentados en la Tabla 5 se observa que la resistencia a la tracción, en todos los casos, superó la mínima especificada en el código ASME IX-13 [13] (620 MPa). Sin embargo, las juntas LDH-A2 y LDH-A4 rompieron en el MS, hecho asociado a defectos internos (escoria atrapada entre la pasada de raíz GTAW y el primer cordón FCAW), mientras que las demás muestras rompieron en la CB. Los valores de elongación de las probetas LDH-A2 y A4 fueron de 7,3 y 8,3\% respectivamente, evidenciando una rotura defectuosa. No se encontraron grandes variaciones en los promedios de esta propiedad para las distintas condiciones analizadas.

\subsection{Ensayos de Charpy-V}

En las Tablas 6 y 7 se puede observar los resultados de energía absorbida por las muestras en las diferentes condiciones, con las entallas ubicadas tanto en el MS como a $2 \mathrm{~mm}$ de la línea de fusión. En todos los casos, los valores de tenacidad cumplieron con las especificaciones mínimas establecidas en el código para la energía absorbida y la expansión lateral [18].

Tabla 6. Valores de Energía obtenidos.

\begin{tabular}{|c|c|c|c|c|c|c|c|c|}
\hline \multirow[b]{2}{*}{ Cupón } & \multicolumn{4}{|c|}{ Metal de Soldadura } & \multicolumn{4}{|c|}{ ZAC } \\
\hline & E1 & E2 & E3 & $\begin{array}{c}\text { E-promedio } \\
\text { (J) }\end{array}$ & E1 & E2 & E3 & $\begin{array}{c}\text { E-promedio } \\
\text { (J) }\end{array}$ \\
\hline DH & 23,5 & 24,9 & 25,4 & 24,6 & 75,2 & 81,3 & 59,3 & 71,9 \\
\hline DL & 20,5 & 21,4 & 22,4 & 21,4 & 65,3 & 70,4 & 77,4 & 71,0 \\
\hline LDH & 23,8 & 20,5 & 23,7 & 22,7 & 38,3 & 25,9 & 60,4 & 41,5 \\
\hline LDL & 21,9 & 23,0 & 21,7 & 22,2 & 43,7 & 55,2 & 34,2 & 44,4 \\
\hline
\end{tabular}

Tabla 7. Valores de expansión lateral obtenidos.

\begin{tabular}{|c|c|c|c|c|c|c|c|c|}
\hline \multirow[b]{2}{*}{ Cupón } & \multicolumn{4}{|c|}{ Metal de Soldadura } & \multicolumn{4}{|c|}{ ZAC } \\
\hline & Exp.1 & Exp.2 & Exp.3 & $\begin{array}{c}\text { Exp-promedio } \\
\text { (mm) }\end{array}$ & Exp.1 & Exp.2 & Exp.3 & $\begin{array}{l}\text { Exp-promedio } \\
(\mathrm{mm})\end{array}$ \\
\hline DH & 0,65 & 0,69 & 0,68 & 0,67 & 1,87 & 1,94 & 1,62 & 1,81 \\
\hline DL & 0,54 & 0,53 & 0,56 & 0,54 & 1,61 & 1,83 & 1,90 & 1,78 \\
\hline LDH & 0,66 & 0,65 & 0,58 & 0,44 & 0,93 & 0,80 & 1,48 & 1,07 \\
\hline LDL & 0,66 & 0,55 & 0,58 & 0,60 & 1,10 & 1.43 & 1,19 & 0,76 \\
\hline
\end{tabular}

Los valores promedios de tenacidad en el MS fueron de 24,6; 21,$4 ; 22,7$ y $22,2 \mathrm{~J}$, para los cupones DH, DL, LDH y LDL, respectivamente (mínimo requerido $20 \mathrm{~J}$ ). Se puede apreciar que no hubo grandes variaciones de la tenacidad en metal de soldadura para las diferentes condiciones estudiadas. Sin embargo, la tenacidad a la fractura de los cupones soldados con consumibles DSS fue levemente mayor que los soldados con consumibles LDSS. En este sentido, los menores valores de ferrita o mayores valores de austenita, medidos en el MS para los consumibles DSS, podrían explicar estos resultados ya que al aumentar el contenido de austenita en la microestructura aumentan los valores de tenacidad [10]. Además, los cupones soldados con menores calores aportados presentaron los menores valores de tenacidad para ambos consumibles. El alto aporte térmico favorece la presencia de austenita (primaria y secundaria discutida anteriormente) justificando estas observaciones [10]. Resultados similares fueron encontrados en los valores de expansión lateral.

Como parte del estudio de las juntas soldadas, se determinó la resistencia al impacto Charpy-V en las ZACs de las mismas, esperando obtener resultados similares dado que los procedimientos de soldadura fueron idénticos para ambos consumibles (alto y bajo calor aportado). 
El análisis de los resultados promedio de tenacidad, obtenido en la ZAC muestra grandes diferencias en los consumibles empleados. Sin embargo, no se observaron modificaciones respecto del calor aportado. Para entender e interpretar los resultados obtenidos en la ZAC se recurrió al análisis de la superficie de fractura de las probetas de Charpy-V, identificando la dirección del avance de la fractura y las zonas en ella involucradas. Como se mostró anteriormente en la Figura 4, la ZAC en estas juntas es muy angosta $(150 \mu \mathrm{m})$ por lo que la fractura en su avance podrá hacerlo hacia el MS (de menor tenacidad) o al CB (de mayor tenacidad). De esta manera la tenacidad medida se verá afectada por la proporción de las mencionadas zonas involucradas. La Figura 7 muestra la zona por donde se propagó la fractura de la muestra LDL de 34,2 J en una de las caras. En esta imagen se puede observar claramente que la fractura avanzó sobre el MS. Sin embargo, en la Figura 8 se puede observar que la fractura avanzó fundamentalmente por la cañería base. Por consiguiente la energía absorbida dependerá de la proporción de estas zonas. Esto justificaría la dispersión encontrada en los resultados de impacto Charpy -V en las ZACs.

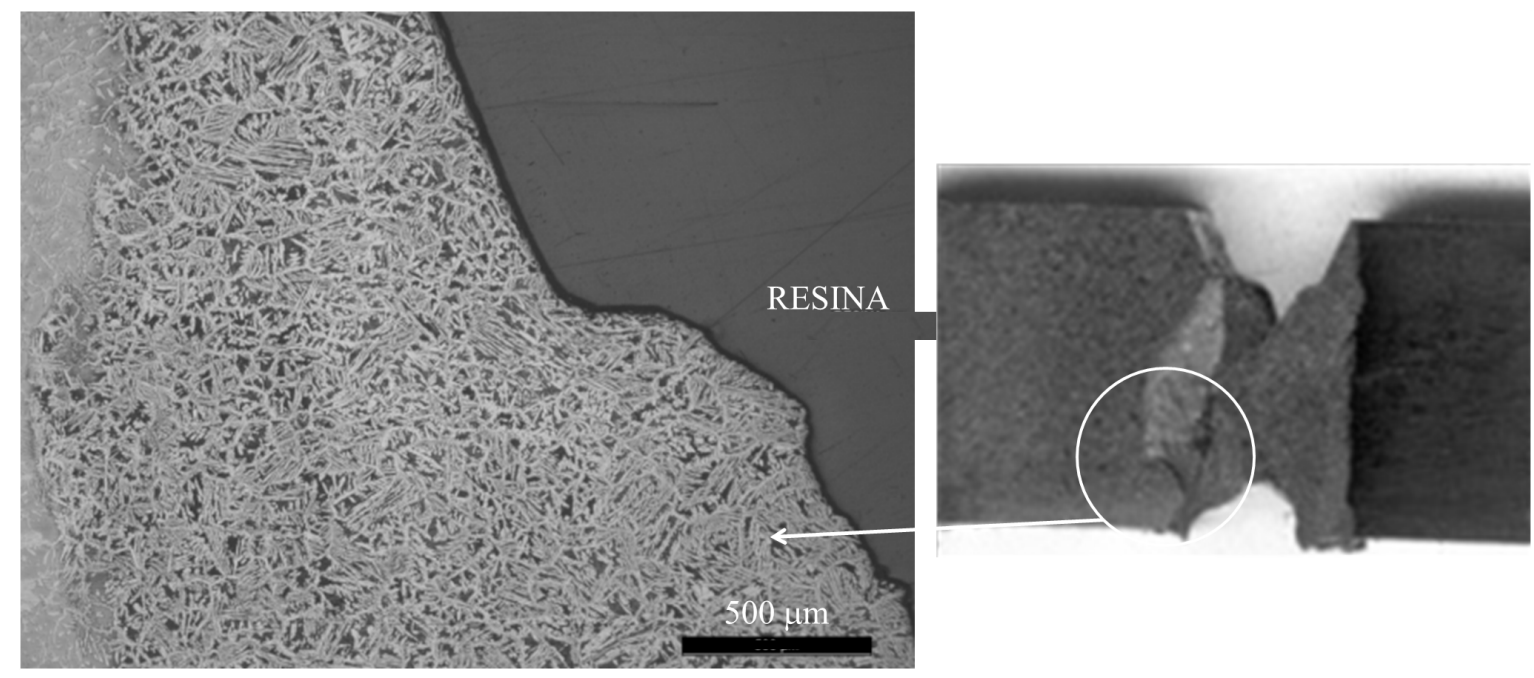

Figura 7. Microestructura en un lado de la superficie de fractura de la probeta $L D L E=34,2 \mathrm{~J}$. La fractura avanza en el MS.

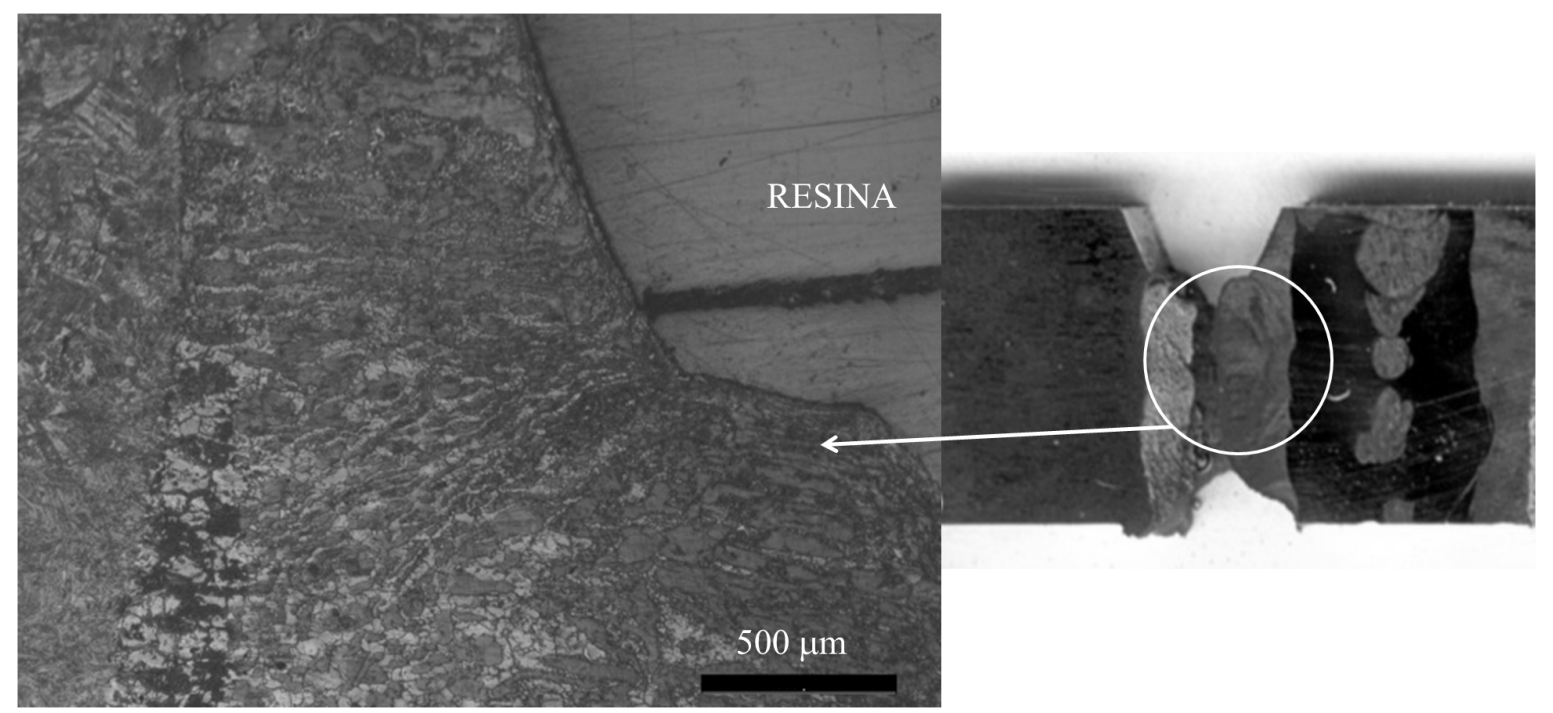

Figura 8. Microestructura en el otro lado de la superficie de fractura de la probeta LDL E=34,2 J. La fractura avanza en el metal base (CB). 
Efecto de la Composición Química del Metal de Aporte y del Calor Aportado Sobre la Microestructura y las Propiedades Mecánicas de Juntas Soldadas de Aceros Inoxidables Dúplex

\section{Conclusiones}

Las soldaduras de cañerías de acero inoxidable dúplex, con pasada de raíz GTAW-DSS y relleno FCAW con consumibles DSS y LDSS, soldados con alto y bajo calor aportado, generaron las siguientes conclusiones:

Para todas las condiciones:

1) La microestructura estuvo constituida en la ZAC por dos fases, ferrita y austenita y en el MS se observó la presencia de austenita secundaria. Sin embrago, el tamaño, la morfología y el contenido de las fases cambió dependiendo de la zona de observación.

2) La ZAC presentó mayores contenidos de ferrita con austenita intergranular. El ancho de la ZAC fue de aproximadamente $150 \mu \mathrm{m}$.

3)Se encontró austenita secundaria en las zonas recristalizadas entre cordones del MS.

4) No se encontraron diferencias significativas entre las mediciones de ferrita por medio de un ferritómetro y por microscopia óptica.

5)En los valores de dureza no se encontraron grandes variaciones en las diferentes zonas analizadas (CB, ZAC y MS). Se notó un muy leve incremento de dureza en la ZAC de la cañería base. Se observó que la dureza de la pasada de raíz (GTAW) fue levemente mayor que la de los cordones de relleno (FCAW).

6) La resistencia a la tracción, en todos los casos, superó lo especificado.

7)En el MS los valores de energía absorbida y expansión lateral superaron lo especificado.

8) La tenacidad en el MS fue mayor en los cupones con menores contenidos de ferrita.

9) La energía absorbida en la ZAC es dependientes de la zona de propagación de la fractura y no es un valor representativo de la tenacidad en esa zona.

Para DSS y LDSS, alto y bajo calor aportado:

1) Las juntas soldadas con consumibles FCAW DSS tuvieron leves menores contenidos de ferrita, respecto de los consumibles FCAW LDSS. Además, se observaron leves mayores contenidos de ferrita en las condiciones soldadas con menor aporte térmico.

2) No se encontraron grandes variaciones en los promedios generales de dureza para las distintas condiciones analizadas. Sin embargo, las probetas soldadas con menor aporte térmico tuvieron una leve mayor dureza.

3) No se encontraron grandes variaciones en los promedios de la resistencia a la tracción para las distintas condiciones analizadas. Sin embargo, las probetas soldadas con menor aporte térmico tuvieron resistencias superiores a las obtenidas con bajo aporte térmico.

4) Los valores de tenacidad en el MS fueron similares para ambos consumibles.

Comentario adicional: En nuestra industria nacional, por un tema de disponibilidad o acceso se utilizan los materiales bases más vendidos o populares (en este caso, los aceros inoxidables dúplex), incluso en aplicaciones donde no es necesario hacerlo y donde se podrían utilizar aceros inoxidables lean dúplex (de menor acceso). En estas condiciones, la soldadura de cañerías de aceros inoxidables dúplex (únicas disponibles) podrían soldarse con consumibles lean dúplex abaratando los costos. Esto está justificado porque ambos consumibles satisfacen los requerimientos de esta aplicación. Es decir, en aquellas condiciones de servicio de baja agresividad, en que pueda utilizarse cañerías de aceros lean dúplex, pero a falta de ellas, las de aceros inoxidables dúplex podrían soldarse con consumibles más económicos, como los lean dúplex, ya que cumplen con los requerimientos mecánicos y se disminuye los costos de la obra. 


\section{Agradecimientos}

Los autores agradecen a BOHELER Argentina por la provisión de los consumibles utilizados, a CRECER INGENIERÍA Y CONSTRUCCIONES S.A. por la donación de los caños de DSS, a ESAB-CONARCO por la realización de los análisis químicos, al LABORATORIO DE MICROSCOPÍA ELECTRÓNICA DE INTI - MECÁNICA por la realización de la microscopía electrónica de barrido y a ANPCyT por el apoyo económico al presente proyecto.

\section{Referencias}

[1] Garzón CM, Ramirez AJ. Growth kinetics of secondary austenite in the welding microstructure of a UNS S32304 duplex stainless steel. Acta Materiala. 2006;54(12):3321-3331.

[2] Huang CS, Shih CC. Effects of nitrogen and high temperature aging on sigma phase precipitation of duplex stainless steel. Materials Science and Engineering A. 2005;402(1):66-75. http:// dx.doi.org/10.1016/j.msea.2005.03.111.

[3] Escriba DM, Materna-Morris E, Plaut RL, Padilha AF. Chi-phase precipitation in a duplex stainless steel. Materials Characterization. 2009;60(11):1214-1219. http://dx.doi. org/10.1016/j.matchar.2009.04.013.

[4] Angelini E, Benedetti B, Rosalbino F. Microstructural evolution and localized corrosion resistance of an aged superduplex stainless steel. Corrosion Science. 2004;46(6):1351-1367. http:// dx.doi.org/10.1016/j.corsci.2003.09.024.

[5] Karlsson L. Welding duplex stainless steels: a review of current recommendations. Welding in the World. 2011;56(5):65-76.

[6] Zanotto F, Grassi V, Balbo A, Monticelli C, Zucchi F. Stress corrosion cracking of LDX 2101 duplex stainless steel in chloride solutions in the presence of thiosulphate. Corrosion Science. 2014;80:205-212. http://dx.doi.org/10.1016/j.corsci.2013.11.028.

[7] Liou HY, Hsieh RI, Tsai WT. Microstructure and stress corrosion cracking in simulated heat-affected zones of duplex stainless steels. Corrosion Science. 2002;44(12):2841-2856. http://dx.doi. org/10.1016/S0010-938X(02)00068-9.

[8] Liou HY, Hsieh RI, Tsai WT. Microstructure and pitting corrosion in simulated heat-affected zones of duplex stainless steels. Materials Chemistry and Physics. 2002;74(1):33-42. http:// dx.doi.org/10.1016/S0254-0584(01)00409-6.

[9] Pardal JM, Tavares SSM, Fonseca MC, Souza JA, Côrte RRA, Abreu HFG. Influence of the grain size on deleterious phase precipitation in superduplex stainless steel UNS S32750. Materials Characterization. 2009;60(3):165-172. http://dx.doi. org/10.1016/j.matchar.2008.08.007.

[10] Lippold JC, Kotecki DJ. Welding metallurgy and weldability of stainless steels. Hoboken: Wiley-Interscience; 2005.

[11] American Welding Society. A5.22-95: E2209T1. BÖHLER CN22/9 PW-FD: GMAW flux cored wire, high-alloyed, highly corrosion resistant. Miami: AWS; 1995.
[12] American Welding Society. A5.22-95: E2209T1-G. BÖHLER CN24/9 LDX-PW-FD: GMAW flux cored wire, high-alloyed, highly corrosion resistant. Miami: AWS; 1995.

[13] American Society of Mechanical Engineers. PD190 - BPV Code: section IX: welding, brazing, and fusing qualifications. New York: ASME; 2013.

[14] American Society of Mechanical Engineers. BPVC-IIC - 2013 BPVC section II: materials-Part C: specifications for welding rods, electrodes, and filler metals. New York: ASME; 2013.

[15] American Society for Testing Materials. E562-02: standard test method for determining volume fraction by systematic manual point count. West Conshohocken: ASTM; 2002.

[16] American Society for Testing Materials. E384-07: standard test method for microindentation hardness of materials. West Conshohocken: ASTM; 2007.

[17] American Society for Testing Materials. E23-03: standard test methods for notched bar impact testing of metallic materials. West Conshohocken: ASTM; 2003.

[18] American Society of Mechanical Engineers. VIII Div. 1: rules for construction of pressure vessels. New York: ASME; 2013.

[19] American Society for Testing Materials. G48-03: standard test methods for pitting and crevice corrosion resistance of stainless steels and related alloys by use of ferric chloride solution. West Conshohocken: ASTM; 2003.

[20] Paladino M. Caracterización de soldadura de acero inoxidable dúplex en tubos ASTM A790 tipo UNS S31803 [doctor thesis]. Neuquén: Universidad Nacional del COMAHUE; 2013. $96 \mathrm{f}$.

[21] Kordatos JD, Fourlaris G, Papadimitriou G. The effect of cooling rate on the mechanical and corrosion properties of SAF 2205 (UNS 31803) duplex stainless steel welds. Scripta Materialia. 2001;44(3):401-408. http://dx.doi.org/10.1016/ S1359-6462(00)00613-8.

[22] Muthupandi V, Bala Srinivasan P, Shankar V, Seshadri SK, Sundaresan S. Effect of nickel and nitrogen addition on the microstructure and mechanical properties of power beam processed duplex stainless steel (UNS 31803) weld metals. Materials Letters. 2005;59(18):2305-2309. http://dx.doi. org/10.1016/j.matlet.2005.03.010.

[23] Muthupandi V, Bala Srinivasan P, Seshadri SK, Sundaresan S. Effect of weld metal chemistry and heat input on the structure and properties of duplex stainless steel welds. Materials Science and Engineering A. 2003;358(1-2):9-16. http://dx.doi. org/10.1016/S0921-5093(03)00077-7. 